\title{
artigo
}

Maciel, C.T.; Mesquita, M.G.R.; Paes, G.O.; Stipp, M.A.C.; Trotte, L.A.C.

Estratificação de risco cardiovascular em usuários na Estratégia de Saúde da Familia: uma ferramenta para o cuidado

\section{Estratificação de risco cardiovascular em usuários na Estratégia de Saúde da Família: uma ferramenta para o cuildado}

\author{
Stratification of cardiovascular risk in users in the Family Health Strategy: a tool for care
}

Estratificación del riesgo cardiovascular en los usuarios en la Estrategia de salud familiar: una herramienta para la atención

\begin{abstract}
RESUMO
Objetivo: Caracterizar tipos de risco cardiovascular nos pacientes hipertensos atendidos por uma equipe de saúde da família do município do Rio de Janeiro através do escore de Framingham. Métodos: Estudo observacional, transversal, realizado em uma Clínica de Saúde da Família no município do Rio de Janeiro, no mês de outubro de 2017, através de consulta a prontuários dos pacientes hipertensos cadastrados. Os dados foram categorizados em um banco de dados no programa Excel® 2013 e realizado testes estatísticos descritivos. Resultados: Foram obtidos dados de 221 indivíduos e a maioria dos participantes foram considerados de alto e médio risco para desenvolvimento de evento cardiovascular maior em 10 anos. Conclusão: 0 Escore de Risco de Framingham foi uma ferramenta exitosa para a estratificação de risco de usuários na Atenção Primária à Saúde e merece ser incorporado como instrumento pela equipe de saúde na promoção da saúde cardiovascular.
\end{abstract}

DESCRITORES: Atenção Primária à Saúde; Hipertensão; Doenças Cardiovasculares.

\section{ABSTRACT}

Objective: To characterize types of cardiovascular risk in hypertensive patients treated by a family health team in the city of Rio de Janeiro using the Framingham score. Methods: Observational, cross-sectional study, carried out at a Family Health Clinic in the city of Rio de Janeiro in October 2017, through consultation of medical records of registered hypertensive patients. The data were categorized in a database in the Excel ${ }^{\circledR} 2013$ program and descriptive statistical tests were performed. Results: Data were obtained from 221 individuals and most participants were at high and medium risk for developing a major cardiovascular event in 10 years. Conclusion: The Framingham Risk Score was a successful tool for stratifying the risk of users in Primary Health Care and deserves to be incorporated as an instrument by the health team in promoting cardiovascular health.

DESCRIPTORS: Primary Health Care; Hypertension; Cardiovascular Diseases.

\section{RESUMEN}

Objetivo: caracterizar los tipos de riesgo cardiovascular en pacientes hipertensos tratados por un equipo de salud familiar en la ciudad de Río de Janeiro utilizando el puntaje Framingham. Métodos: Estudio observacional, transversal, realizado en una Clínica de Salud Familiar en la ciudad de Río de Janeiro, en octubre de 2017, a través de la consulta de registros médicos de pacientes hipertensos registrados. Los datos se clasificaron en una base de datos en el programa Exce| ${ }^{\circledR} 2013$ y se realizaron pruebas estadísticas descriptivas. Resultados: se obtuvieron datos de 221 individuos y se consideró que la mayoría de los participantes tenían un riesgo alto y medio de desarrollar un evento cardiovascular mayor en 10 años. Conclusión: El Framingham Risk Score fue una herramienta exitosa para estratificar el riesgo de los usuarios en Atención Primaria de Salud y merece ser incorporado como un instrumento por el equipo de salud para promover la salud cardiovascular. DESCRIPTORES: Atención Primaria de Salud; Hipertensión; Enfermedades Cardiovasculares.

RECEBIDO EM: 31/01/2020 APROVADO EM: 02/02/2020

\section{Caroline Teixeira Maciel}

Enfermeira. Especialista em Saúde da Família e Comunidade pela Escola de Enfermagem Anna Nery da Universidade Federal do Rio de Janeiro. https://orcid.org/0000-0002-6897-1212

\section{Maria Gefé da Rosa Mesquita}

Enfermeira. Doutora em Enfermagem. Professor Adjunto do Departamento de Metodologia da Enfermagem da Escola de Enfermagem Anna Nery da Universidade Federal do Rio de Janeiro. https://orcid.org/0000-0003-3544-347X 


\section{Graciele Oroski Paes}

Enfermeira. Pós-Doutora em Enfermagem. Professor Associado do Departamento de Enfermagem Fundamental da Escola de Enfermagem Anna Nery da Universidade Federal do Rio de Janeiro. https://orcid.org/0000-0001-8814-5770

\section{Marluci Andrade Conceição Stipp}

Pós-Doutora em Enfermagem. Professor Titular do Departamento de Metodologia da Enfermagem da Escola de Enfermagem Anna Nery da Universidade Federal do Rio de Janeiro. https://orcid.org/0000-0002-9534-6324

\section{Liana Amorim Corrêa Trotte}

Enfermeira. Doutora em Enfermagem. Professor Adjunto do Departamento de Metodologia da Enfermagem da Escola de Enfermagem Anna Nery da Universidade Federal do Rio de Janeiro. https://orcid.org/0000-0002-6579-7108

\section{INTRODUÇÃO}

0 Sistema Único de Saúde (SUS) vem sendo aperfeiçoado e avançou em decorrência da implantação do Programa de Saúde da Família (PSF), em 1994, transformando o modelo assistencial, através da incorporação de práticas inovadoras voltadas para a família e comunidade. Renomeado, em 2003, como Estratégia Saúde da Família (ESF), é considerada uma estratégia de expansão e qualificação da atenção básica por fortalecer a premissa de levar a saúde para perto das famílias e ser a porta de entrada nos ser$\operatorname{viços}^{(1)}$. A atenção básica à saúde possui como premissa ser a porta de entrada da população na busca ao acesso universal em saúde, além de ser também uma ferramenta de proteção social em saúde. Somente ela é capaz de produzir e devolver respostas que se aproximem da realidade local do usuário e com isso contribuir diretamente na mudança de estilos de vida, além de, ser o principal ator na construção e coordenação de uma resposta ao cuidado da população em todos níveis de atenção ${ }^{(2)}$.

A ESF, dentre os diversos campos de atuação da saúde, oportuniza um espaço privilegiado para a aplicação diferenciada das práticas de cuidado. Através dela, é possível identificar de modo aproximado o contexto da população as suas principais demandas e, com isso, busca desenvolver uma assistência eficaz, impactando positivamente nas condições de saúde da população, neste caso destacam-se as Doenças Crônicas não Transmissíveis - $\mathrm{DCNT}^{(3)}$.

As DCNT são consideradas um problema de saúde pública, pois são doenças multifatoriais que se desenvolvem no de- correr da vida e tem longa duração. Mundialmente, a hipertensão arterial sistêmica (HAS) se destaca como a doença mais prevalente, responsável por $13,5 \%$ de todas as mortes, aumentando significativamente o risco cardiovascular. No Brasil, sua prevalência média da população em geral é de $32,5 \%(4,5)$.

Segundo os dados da Vigilância de Fatores de Risco e Proteção para Doenças Crônicas por Inquérito Telefônico (Vigitel) 2018, a frequência de diagnósticos de hipertensão aumentou com a idade e diminuiu com o nível de escolaridade, em ambos os sexos, tendo o Rio de Janeiro um percentual de $31,2 \%^{(6)}$. A Hipertensão Arterial (HA) é caracterizada por elevação sustentada dos níveis pressóricos $\geq 140$ e/ ou $90 \mathrm{mmHg}$. Frequentemente se associa a distúrbios metabólicos, alterações funcionais e/ou estruturais de órgãos-alvo, sendo agravada pela presença de outros fatores de risco (FR), como: o tabagismo, sedentarismo e dieta rica em gordura, dislipidemia, obesidade abdominal, intolerância à glicose e diabetes mellitus - $\mathrm{DM}^{(7)}$.

Estudos apontam que a HAS é um dos principais fatores de risco para o desenvolvimento das Doenças Cardiovasculares (DCV), tais como: doença arterial coronariana, insuficiência cardíaca, doença cerebrovascular, doença renal crônica e fibrilação atrial. Embora a HAS também seja uma doença cardiovascular, apresenta-se aqui em destaque por sua alta prevalência, cronicidade, baixo controle e elevado custo sócio financeiro ${ }^{(8)}$.

A partir da medicina baseada em evidência, algoritmos foram criados para estratificação de risco de doenças, sendo estes de simples realização e de baixo custo.
Dentre tais algoritmos, destaca-se o Escore de Risco de Framingham (ERF), sendo este uma ferramenta que avalia o risco de doença cardiovascular, de acordo com a presença ou não de certos fatores de risco. O ERF é um instrumento utilizado para o cálculo e estimativa de risco de evento cardiovascular absoluto na prática clínica, classificando o indivíduo em baixo, médio e alto risco, sendo assim capaz de estimar o risco de desenvolvimento de DCV em um período de dez anos. Mundialmente reconhecido e amplamente aplicado, este possibilita o ajuste entre a intensidade do fator de risco e a probabilidade estimada da doença, tornando o tratamento mais custo-efetivo $^{(9)}$.

Sendo uma importante ferramenta para o cuidado do usuário com HAS, essa estratificação do risco cardiovascular pelo ERF deve ser realizada na ESF, desempenhando o papel de norteador da linha de cuidado do usuário, de forma a orientar o percurso desse usuário dentro da rede de atenção à saúde, sendo ora na Atenção Primária à Saúde (APS), ora em Atenção Ambulatorial Especializada, de acordo com os resultados do escore ${ }^{(4,10)}$.

De acordo com a pontuação revelada pelo ERF, a rotina de consultas com os profissionais de saúde ocorre de forma distinta, ou seja, um usuário classificado como baixo risco, de até $10 \%$ risco de eventos cardiovasculares em indivíduos sem doença aterosclerótica, indica-se consultas anuais com o médico e com o enfermeiro. Para aqueles com risco moderado, entre $10 \%$ a $20 \%$, deverão ser realizadas consultas médicas e de enfermagem semestralmente. Já para os pacientes classificados como alto risco, que significa uma chance de $20 \%$ ou 
mais de desenvolver um evento cardiovascular maior farão acompanhamento médico e de enfermagem quadrimestral ${ }^{(11)}$.

Enquanto profissionais inseridos em uma equipe do ESF que atendem um número importante de usuários com HAS e diante da problemática exposta, interroga-se: Qual é o risco cardiovascular de acordo com a ERF de o usuário hipertenso em atendimento em uma unidade básica de saúde desenvolver um evento cardiovascular maior? E para responder esta questão norteadora foi operacionalizado o seguinte objetivo: caracterizar tipos de risco cardiovascular nos pacientes hipertensos atendidos por uma equipe de saúde da família do município do Rio de Janeiro através do escore de Framingham.

\section{METODOLOGIA}

Trata-se de um estudo observacional, transversal, realizado em uma Clínica de Saúde da Família, no Complexo do Alemão, no município do Rio de Janeiro. Esta unidade possui um total de 15 equipes, tendo uma área de cobertura de 46.000 pacientes. Optou-se pela análise apenas de pacientes de uma equipe pré-estabelecida, o motivo da escolha se deu por um dos pesquisadores estar alocado como enfermeira desta equipe durante o período de coleta de dados. A equipe pesquisada tem uma população adstrita de 2845 pacientes, sendo 590 hipertensos, 164 diabéticos, 200 crianças, 752 mulheres e 1139 pacientes que não se enquadram em nenhum desses grupos. Os pesquisadores optaram por uma amostragem de conveniência devido ao pequeno período de tempo para realização da coleta de dados, desta forma, tentou-se garantir o maior número possível de participantes para realização do estudo.

Os critérios de inclusão utilizados foram: pacientes hipertensos da equipe, maiores de 18 anos, de ambos os sexos e que possuíam o registro do seguintes itens no prontuário eletrônico: sexo, idade, níveis pressóricos, prescrição médica de anti-hipertensivo, história coletada e documentada de presença ou não de tabagismo presente ou passada e ou presença

Trata-se de um estudo

\section{observacional,}

transversal,

realizado em

uma Clínica de

Saúde da Família,

no Complexo

do Alemão, no

município do

Rio de Janeiro.

Esta unidade

possui um total

de 15 equipes,

tendo uma área

de cobertura de

46.000 pacientes. ou não atual de diabetes, além de níveis de colesterol total e HDL (High Density Lipoproteins) colesterol. Os critérios de exclusão foram pacientes cujos prontuários não possuíssem dados atualizados (últimos 6 meses) dos seguintes itens: níveis pressóricos, prescrição médica de anti-hipertensivo e níveis de colesterol total e HDL colesterol.

A coleta de dados foi realizada no mês de outubro de 2017, através de uma lista pré-estabelecida emitida pelo prontuário eletrônico, no qual foi enumerado todos os pacientes hipertensos da equipe. Após, a pesquisadora aplicou os critérios de inclusão e exclusão sendo selecionados para este estudo 221 participantes que completaram todos os critérios. Em seguida foi calculado o Escore de Framingham utilizando o aplicativo Calculadora de Risco Cardiovascular desenvolvido pelo núcleo de telessaúdeRS/UFRGS (Universidade Federal do Rio Grande do Sul) para o Ministério de Saúde do Brasil, que foi baseado no estudo de D'Agostino e colaboradores ${ }^{(10)}$.

Os dados foram digitados, categorizados e codificados em um banco de dados no programa Excel $^{\circ} 2013$, do pacote Microsoft $^{\circ}$ e, através do mesmo, foi realizado testes estatísticos descritivos. A normalidade da amostra foi verificada pelo teste de Kolmogorov-Smirno e a apresentação dos dados se deu em números absolutos, percentagens e ou frequências, média e desvio-padrão.

Por se tratar de uma pesquisa envolvendo seres humanos, o projeto foi apreciado por dois comitês de éticas de em pesquisa (CEPs) e aprovados conforme pareceres n. ${ }^{\circ}$ 2.312.534 (Universidade Federal do Rio de Janeiro) e n. ${ }^{\circ} 2.312 .534$ (Secretaria Municipal de Saúde da cidade do Rio de Janeiro). Devido a impossibilidade de contatar todos os participantes da pesquisa em um período de 01 mês (tempo da coleta de dados), assim como, conseguir a presença de todos durante este período para obtenção do Termo de Consentimento Livre e Esclarecido (TCLE), houve entendimento pelos membros dos CEPs sobre a dispensa do TCLE, e a garantia dos participantes foi preservada através da 
concessão do Termo de Confidencialidade entregues aos CEPs pelos pesquisadores.

\section{RESULTADOS}

Foram obtidos dados de 221 indivíduos dos 590 que possuíam o diagnóstico de hipertensão. Na amostra estudada, houve predomínio do sexo feminino e observou-se que os valores de níveis pressóricos estavam fora da faixa apropriada para controle apesar do uso de anti-hipertensivo

\section{Tabela 1. Caracterização da amostra. Rio de Janeiro, RJ, Brasil, 2017.}

VARIÁVEIS

\section{SEXO}

Feminino

Masculino

IDADE

\section{DIABETES MELLITUS}

Sim

Não

TABAGISMO

Sim

Não

PAS (pressão arterial

sistólica)

PAD (pressão arterial

diastólica)

COLESTEROL

Total

$\mathrm{HDL}$

RISCO ALTO\$

Homem

Mulher

Total

RISCO MÉDIO\$

Homem

Mulher

Total

RISCO BAIXO\$

Homem

Mulher

Total

Nota: Número total de indivíduos $=221 ;{ }^{*}$ méd

Framingham por D’Agostino e colaboradores. por todos. O colesterol total estava muito pouco acima do limite e o HDL colesterol estava dentro da faixa com tendência para valores mais próximos dos limites inferiores. A maioria dos participantes foram considerados de alto e médio risco para desenvolvimento de evento cardiovascular maior em 10 anos. A características da amostra estão detalhadas na Tabela 1 .

\section{DISCUSSÃO}

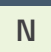

80

141

27

$\%$

$66 \%$

$34 \%$

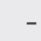

$36 \%$

$64 \%$

$12 \%$

$88 \%$

194
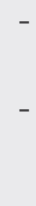

43

45

88

22

51

73

11

29

60
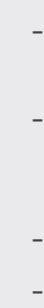

$20 \%$

$20 \%$

$40 \%$

$10 \%$

$23 \%$

$33 \%$

$5 \%$

$13 \%$

$27 \%$
DESVIO PADRÃO

$58^{*}(10)^{\#}$

$85^{*}(12)^{\#}$

$209 *(46)^{\#}$

$52^{*}(18)^{\#}$
Os resultados deste estudo mostram que na população hipertensa avaliada houve uma presença de outros fatores de risco que aumentam a probabilidade de desenvolvimento de um evento cardiovascular maior em 10 anos. Este achado corrobora com outros estudos nacionais e internacionais que associam tais resultados como consequência de hábitos de vida da pessoa, a má adesão à terapêutica prescrita e atividade física inativa, aumentando assim o risco de morbidade e mortalidade ${ }^{(12,13)}$.

A maioria da amostra estudada, composta por usuários atendidos pela equipe da ESF, foram classificados em risco cardiovascular alto ou moderado. Tal achado ressalta o desafio encontrado no cotidiano da Atenção Primária à Saúde no que se refere à articulação do cuidado com as condições de saúde e a capacidade de resposta às necessidades do usuário, ou melhor, traçar a linha de cuidado de forma a pactuar os fluxos de atenção, a fim de facilitar o acesso do usuário às Unidades e Serviços que ele necessita, incorporando a integralidade na assistência à saúde ${ }^{(2)}$.

Os dados obtidos referentes ao Diabetes Mellitus destacaram-se por predominarem em um terço da amostra estudada, além de possuir relação direta para risco cardiovascular alto. Tal variável se configura um desafio para a saúde pública nacional e internacional tornando-se imprescindível a construção de ferramentas que facilitem estratégias de prevenção, promoção à saúde e manejo de glicemia na população de risco e demais ${ }^{(14,15)}$.

Apesar do tabagismo não ter sido determinante nesta amostra para determinar a presença de alto ou moderado risco cardiovascular, o fumo é considerado o único fator de risco totalmente evitável para o surgimento das doenças e morte por problemas cardiovasculares. Ressalta-se que a interrupção do hábito de fumar reduz em até $50 \%$ a probabilidade de DCV, porém, mesmo em indivíduos que deixaram de fumar não se sabe exatamente o grau da lesão já existente ${ }^{(16)}$.

Em relação à HAS, o valor médio de pressão arterial sistólica e diastólica dos usuários encontrou-se elevada, tendo em 
vista que todos os pacientes da amostra fazem uso de medicação anti-hipertensiva. A estratificação da hipertensão é uma forma de adotar melhores estratégias no manejo da mesma, conforme o risco projetado para o desenvolvimento de um evento cardiovascular. As duas principais diretrizes de prevenção e controle da HAS, das associações europeia e americana de cardiologia concordam que o controle da pressão arterial deve acontecer no domicílio e no ambulatório e que médicos devem fazer uso restrito de betabloqueadores como terapia de primeira linha. Além disso, ambas recomendam que pacientes identificados como alto risco cardiovascular devem ter metas de níveis pressóricos mais baixos e um tratamento anti-hipertensivo mais intensivo ${ }^{(17)}$.

Dentre fatores que contribuem para adesão ao tratamento da HAS em pacientes da atenção primária, foi observado que renda familiar mais alta e presença de companheiro (união estável ou casamento) eram protetores. Já em idosos, os principais fatores de risco para má adesão eram: idade mais avançada, menor escolaridade, sexo masculino e raça/cor não branca(18). Também foi observado em outra pesquisa que pacientes que afirmavam realizar o tratamento para HAS apenas faziam uso do tratamento medicamentoso, indicando falta de conhecimento de medidas de tratamento não farmacológicas ou falha dos profissionais de saúde da ESF em sensibilizar os pacientes para a importância destas medidas terapêuticas ${ }^{(19)}$. Ademais, vários fatores podem dificultar a adesão ao tratamento da hipertensão, como baixa condição econômica, grau de conhecimento sobre doença, dificuldade de acesso aos serviços de saúde, ausência de sintomas físicos que demonstrem aumento da pressão arterial e cronicidade da doença ${ }^{(20)}$.

Os dados referentes ao perfil lipídico dos sujeitos estudados à luz das recomendações da atualização da diretriz brasileira para o controle da dislipidemia e prevenção de aterosclerose ${ }^{(21)}$ não é alarmante, já a média do colesterol total encontra-se na categoria classificada como limítrofe para a população acima de 20 anos, embora esse valor não seja classificado como alto, deve ser otimizado para atingir a categoria desejável, abaixo de $190 \mathrm{mg} / \mathrm{dl}$. De acordo com a literatura, o colesterol elevado pode ser o principal fator de risco modificável da doença arterial coronariana, sendo o seu controle responsável por diminuir o risco de eventos cardiovasculares como o infarto agudo do miocárdio. As diretrizes atuais de prevenção dos EUA para controle da pressão arterial e do colesterol recomendam o uso das equações de coorte combinadas para iniciar um processo de tomada de decisão compartilhada entre médicos e pacientes na prevenção primária ${ }^{(22)}$.

Para a coordenação e a integração dos serviços e ações de Saúde no atendimento aos usuários deste trabalho, pontuamos que os mesmos se encontram inseridos na Rede de Atenção à Saúde (RAS) das DCNT. As DCNT caracterizam-se não apenas pelo somatório de seus sinais e sintomas em si, mas ainda por seus determinantes sociais, entre eles, as desigualdades sociais, as diferenças no acesso aos bens e aos serviços, a baixa escolaridade, as desigualdades no acesso à informação, além dos fatores de risco modificáveis, como tabagismo, consumo de bebida alcoólica, inatividade física e alimentação inadequada. Resumidamente, a RAS consiste na organização do conjunto de serviços e ações de saúde de distintas densidades tecnológicas que, integrados por meio de estruturas de apoio técnico, logístico e de gestão, buscam garantir a integralidade do cuidado às populações de uma região de saúde $^{(23)}$. Nesse sentido, a APS como porta de entrada dos usuários no sistema de saúde tem papel fundamental para o planejamento e organização da RAS, integrando, coordenando e atendendo aos usuários em suas necessidades de saúde.

Deve-se considerar que quando se propõe estratificar usuários para ajustar seus tratamentos é importante entender que a estratificação não é conduzida apenas pelo número de fatores de risco ou comorbidades, mas também pelo seu tipo. Melhor dizer, hipertensos com diabetes são diferentes de hipertensos tabagistas, o que implica em dizer que o tratamento deve ser adequado a cada usuário, mas não apenas em relação ao número de comorbidades presentes $^{(14)}$. Essas particularidades reforçam a necessidade que os profissionais de saúde inseridos na APS organizem a assistência à saúde desses usuários através de linhas de cuidado traçadas de forma singularizada, de acordo com os resultados das estratificações, fatores de risco identificados e necessidades de saúde apresentadas.

Considerando que a maioria dos usuários foram classificados como risco moderado a alto para desenvolver um evento cardiovascular maior, recomenda-se que a equipe da unidade em questão se reúna a fim de traçar estratégias para otimizar o manejo desses usuários. Uma tecnologia de trabalho em saúde que pode contribuir para uma concepção mais ampliada de saúde e ampliar o conceito das RAS são os "grupos operativos". Criado por Pichon-Rivière e definido como o "conjunto restrito de pessoas, ligadas entre si por constantes de tempo e espaço, articuladas por sua mútua representação interna, que se propõem, de forma explícita ou implícita, a uma tarefa que constitui a sua finalidade", esse conceito pode ser útil como forma de operacionalizar estratégias que permitam que a equipe de saúde e os usuários compartilhem experiências e vivenciem saberes e práticas que facilitam a escuta, o acolhimento e o vínculo ${ }^{(24)}$.

Atualmente, no Brasil, os grupos operativos vêm sendo utilizados em diversas áreas da saúde, especialmente na APS, para promoção de programas educativos em saúde, prevenção de doenças e prestação de cuidados específicos. Como sugestão para a realidade encontrada no estudo, vislumbra-se a criação de um programa de atenção a usuários com risco moderado a alto para desenvolver evento cardiovascular maior, que possibilite progresso no manejo das necessidades de saúde dessas pessoas. Benefícios esperados para tal programa seriam uma maior otimização do trabalho da equipe da unidade na RAS, com o acompanhamento dos hipertensos e diabéticos de forma mais estudada, orientação dos usuários de forma efetiva sobre o perigo de serem classificados como risco moderado a alto para desenvolver um 
evento cardiovascular maior, participação ativa do usuário na construção de seu plano de cuidado e maior envolvimento da equipe de profissionais com o usuário fortalecendo o vínculo.

\section{CONCLUSÃO}

Este trabalho evidenciou que a maioria dos hipertensos acompanhados na atenção primária por uma equipe de estratégia de saúda da família foram classificados pelo Escore de Risco de Framingham como risco moderado e alto. Estes dados afirmam o grande potencial na utilização desta ferramenta de estratificação como instrumento a ser incorporado pela equipe de saúde na promoção da saúde cardiovascular nas consultas realizadas. Todavia, os achados evidenciam que outras ações precisam incor- poradas de forma a considerar o indivíduo hipertenso em todas as suas nuances sociais e culturais, valorizando seu ambiente e suas condutas individuais e coletivas.

Assim, tecnologias educacionais são apresentadas como aliadas no processo de construção de uma visão ampliada do conceito de saúde por usuários. Dentre estas, a tecnologia "Grupos Operativos" pode contribuir como ferramenta a ser incorporada pela equipe como estratégia de promoção do cuidado, uma vez que a mesma abre espaços para a escuta das necessidades das pessoas, possibilitando por meio da dialogicidade, troca de conhecimentos sobre os fatores condicionantes do processo saúde-doença, oferecendo ainda subsídios para a adoção de novos hábitos de saúde. Os grupos operativos na APS permitem que todos possam falar sobre seus problemas e buscar soluções de forma conjunta, fortalecendo o vínculo e promovendo a integralidade.

Por fim, sugere-se a criação de um "Programa de Atenção a Usuários com Risco Moderado a Alto de Evento Cardiovascular Maior" com os usuários da ESF da unidade, de forma a incorporar benefícios da ordem estratégica com a formulação das ações a serem desenvolvidas para atendimento das necessidades de saúde e prevenção de agravos destes e, tático-operacional, com a execução e gestão do programa formulado na unidade e sua interface com as redes de atenção à saúde, de modo a subsidiar a construção de planos terapêuticos articulados, integrais, que considerem as condições e necessidades locais e a autonomia dos usuários nos processos.

\section{REFERÊNCIAS}

1. Cavalcanti PC da S, Oliveira Neto AV de, Sousa MF de. Quais são os desafios para a qualificação da Atenção Básica na visão dos gestores municipais? Saúde em Debate [Internet]. junho de 2015 [citado 30 de janeiro de 2020];39(105):323-36. Disponivel em: http://www.scielo.br/scielo.php?script=sci_arttext\&pi$d=$ S0103-11042015000200323\&lng=pt\&tIng=pt

2. Cecilio LC de O, Reis AAC dos. Apontamentos sobre os desafios (ainda) atuais da atenção básica à saúde. Cadernos de Saúde Pública [Internet]. 20 de agosto de 2018 [citado 30 de janeiro de 2020];34(8). Disponivel em: http://www.scielo.br/scielo.php?script=sci_arttext\&pi$d=S 0102-311 \times 2018000800501 \& \operatorname{lng}=p t \& t \operatorname{lng}=p t$

3. Kebian LVA, Oliveira SA de. <b>Práticas de cuidado de enfermeiros e agentes comunitários de saúde da estratégia saúde da família / Health practices of nurses and community health agents of the family health strategy. Ciência, Cuidado e Saúde [Internet]. 7 de fevereiro de 2015 [citado 30 de janeiro de 2020];14(1):893. Disponível em: http:// periodicos.uem.br/ojs/index.php/CiencCuidSaude/article/ view/22466

4. Centro Universitário Leão Sampaio, da Silva A, Alves dos Santos E, Edilton Alves Feitoza J, de Souza Melo ML, Alves de Oliveira R dos S, et al. Estratégia de educação em saúde para a adesão de hipertensos à consulta de enfermagem na atenção básica. Revista Interfaces: Saúde, Humanas e Tecnologia [Internet]. 23 de julho de 2019 [citado 30 de janeiro de 2020];7(1):203-9. Disponivel em: https://interfaces. leaosampaio.edu.br/index.php/revista-interfaces/article/ view/659/pdf
5. Girão ALA, Freitas CHA de. Usuários hipertensos na atenção primária à saúde: acesso, vínculo e acolhimento à demanda espontânea. Revista Gaúcha de Enfermagem [Internet]. 2016 [citado 30 de janeiro de 2020];37(2). Disponivel em: http://www.scielo.br/scielo.php?script=sci_arttext\&pid=S1983-14472016000200408\&Ing=pt\&tIng=pt

6. Brasil, Ministério da Saúde, Secretaria de Vigilância em Saúde, Departamento de Análise em Saúde e Vigilância de Doenças não Transmissíveis. Vigitel Brasil 2018: vigilância de fatores de risco e proteção para doenças crônicas por inquérito telefônico: estimativas sobre frequência e distribuição sociodemográfica de fatores de risco e proteção para doenças crônicas nas capitais dos 26 estados brasileiros e no Distrito Federal em 2018 [Internet]. Brasília: Ministério da Saúde; 2019 [citado 30 de janeiro de 2020] p. 132. Disponivel em: http://bvsms.saude.gov.br/bvs/publicacoes/vigitel_brasil_2018_vigilancia_fatores_risco.pdf

7. Malachias M, Souza W, Plavnik F, Rodrigues C, Brandão A, Neves M, et al. Capítulo 1 - Conceituação, Epidemiologia e Prevenção Primária. Arquivos Brasileiros de Cardiologia [Internet]. 2016 [citado 30 de janeiro de 2020];107(3). Disponivel em: http://www.gnresearch.org/doi/10.5935/ abc. 20160151

8. Carrington MJ, Stewart S. Cardiovascular disease prevention via a nurse-facilitated intervention clinic in a regional setting: The Protecting Healthy Hearts Program. European Journal of Cardiovascular Nursing [Internet]. agosto de 2015 [citado 30 de janeiro de 2020];14(4):35261. Disponivel em: http://journals.sagepub.com/ doi/10.1177/1474515114537022 


\section{REFERÊNCIAS}

9. Instituto do Coração do Hospital das Clínicas da Faculdade de Medicina da Universidade de São Paulo.São Paulo, SP, Brasil, Marte Chacra AP, Dias dos Santos Filho R. Quando e como avaliar o risco cardiovascular global em indivíduos aparentemente normais - ou check-up para todos. Revista da Sociedade de Cardiologia do Estado de São Paulo [Internet]. 10 de março de 2019 [citado 30 de janeiro de 2020];29(1):46-52. Disponível em: http://socesp.org.br/ revista/edicao-atual/quando-e-como-avaliar-o-risco-cardiovascular-global-em-individuos-aparentemente-normais-ou-check-up-para-todos/103/682/

10. D'Agostino RB, Vasan RS, Pencina MJ, Wolf PA, Cobain M, Massaro JM, et al. General Cardiovascular Risk Profile for Use in Primary Care. Circulation [Internet]. 2008;117(6):743-53. Disponível em: https://www.ahajournals.org/doi/abs/10.1161/CIRCULATIONAHA.107.699579

11. Brasil, Ministério da Saúde, Secretaria de Atenção à Saúde, Departamento de Atenção Básica. Cadernos de Atenção Básica. Estratégias para o cuidado da pessoa com doença crônica: hipertensão arterial sistêmica. Brasília: Ministério da Saúde; 2013. 128 p.

12. Arnett DK, Blumenthal RS, Albert MA, Buroker AB, Goldberger ZD, Hahn EJ, et al. 2019 ACC/AHA Guideline on the Primary Prevention of Cardiovascular Disease: A Report of the American College of Cardiology/American Heart Association Task Force on Clinical Practice Guidelines. Circulation [Internet]. 2019;140(11):e596-646. Disponível em: https://www.ahajournals.org/doi/abs/10.1161/ CIR.0000000000000678

13. Fortuna RJ, Nagel AK, Rose E, McCann R, Teeters JC, Quigley DD, et al. Effectiveness of a multidisciplinary intervention to improve hypertension control in an urban underserved practice. Journal of the American Society of Hypertension [Internet]. dezembro de 2015 [citado 30 de janeiro de 2020];9(12):966-74.Disponivel em: https://linkinghub. elsevier.com/retrieve/pii/S193317111500741X

14. Pimenta HB, Caldeira AP. Fatores de risco cardiovascular do Escore de Framingham entre hipertensos assistidos por equipes de Saúde da Família. Ciência \& Saúde Coletiva [Internet]. junho de 2014 [citado 30 de janeiro de 2020];19(6):1731-9. Disponivel em: http://www.scielo.br/scielo.php?script=sci_arttext\&pi$d=$ S1413-81232014000601731\&Ing=pt\&tIng=pt

15. Sun X, Du T. Trends in cardiovascular risk factors among U.S. men and women with and without diabetes, 19882014. BMC Public Health [Internet]. dezembro de 2017 [citado 30 de janeiro de 2020];17(1). Disponível em: https:// bmcpublichealth.biomedcentral.com/articles/10.1186/ s12889-017-4921-4

16. Malta DC, Stopa SR, Santos MAS, Andrade SSC de A, Oliveira TP, Cristo EB, et al. Evolução de indicadores do tabagismo segundo inquéritos de telefone, 2006-2014. Cadernos de Saúde Pública [Internet]. 21 de setembro de 2017 [citado 30 de janeiro de 2020];33(suppl 3). Disponí- vel em: http://www.scielo.br/scielo.php?script=sci_arttext\&pid=S0102-311X2017001505008\&Ing=pt\&tlng=pt

17. Bakris G, Ali W, Parati G. ACC/AHA Versus ESC/ESH on Hypertension Guidelines. Journal of the American College of Cardiology [Internet]. 2019;73(23):3018-3026. Disponível em: http://www.onlinejacc.org/content/73/23/3018

18. Gewehr DM, Bandeira VAC, Gelatti GT, Colet C de F, Oliveira KR de. Adesão ao tratamento farmacológico da hipertensão arterial na Atenção Primária à Saúde. Saúde em Debate [Internet]. janeiro de 2018 [citado 30 de janeiro de 2020];42(116):179-90. Disponível em: http://www.scielo.br/scielo.php?script=sci_arttext\&pi$\mathrm{d}=$ S0103-11042018000100179\&Ing=pt\&tIng=pt

19. Dias EG, Souza ELS, Mishima SM. Contribuições da Enfermagem na adesão ao tratamento da hipertensão arterial: uma revisão integrativa da literatura brasileira. Revista de Epidemiologia e Controle de Infecção [Internet]. 5 de setembro de 2016 [citado 30 de janeiro de 2020];6(3). Disponível em: https://online.unisc.br/seer/index.php/epidemiologia/article/view/7470

20. Medeiros Filho RDA, Maciel APF, Pimenta HB, Caldeira AP. Prevalence of behaviors and risk factors for cardiovascular diseases in hypertensive population in the north of Minas Gerais, Brazil / Prevalência de comportamentos e fatores de risco para doenças cardiovasculares em população de hipertensos no norte de Minas Gerais, Brasil. Revista de Pesquisa: Cuidado é Fundamental Online [Internet]. 9 de janeiro de 2018 [citado 30 de janeiro de 2020];10(1):90. Disponível em: http://seer.unirio.br/index.php/cuidadofundamental/article/view/5985

21. Faludi A, Izar M, Saraiva J, Chacra A, Bianco $H$, Afiune Neto $A$, et al. Atualização da Diretriz Brasileira de Dislipidemias e prevenção da aterosclerose - 2017. Arquivos Brasileiros de Cardiologia [Internet]. 2017 [citado 30 de janeiro de 2020];109(1). Disponivel em: http://www.gnresearch. org/doi/10.5935/abc.20170121

22. Lloyd-Jones DM, Braun LT, Ndumele CE, Smith SC, Sperling LS, Virani SS, et al. Use of Risk Assessment Tools to Guide Decision-Making in the Primary Prevention of Atherosclerotic Cardiovascular Disease. Journal of the American College of Cardiology [Internet]. junho de 2019 [citado 30 de janeiro de 2020];73(24):3153-67. Disponivel em: https:// linkinghub.elsevier.com/retrieve/pii/S0735109718390363

23. Brasil, Ministério da Saúde, Secretaria de Atenção à Saúde, Departamento de Atenção Básica. Estratégias para o cuidado da pessoa com doença crônica. Brasília: Ministério da Saúde; 2014. 162 p. (Cadernos de Atenção Básica).

24. Menezes KKP de, Avelino PR. Grupos operativos na Atenção Primária à Saúde como prática de discussão e educação: uma revisão. Cadernos Saúde Coletiva [Internet]. março de 2016 [citado 30 de janeiro de 2020];24(1):124-30. Disponível em: http://www.scielo.br/scielo.php?script=sci_arttext\&pid=S1414-462X2016000100124\&Ing=pt\&tIng=pt 